\title{
Design of Financial Accounting Module in Printing Enterprise Based on Web
}

\author{
Peipei Ran ${ }^{1, \text { a }}$, Wenjie Yang ${ }^{1}$, Yuke $\mathrm{Huo}^{1}$ and Shaozhong $\mathrm{Cao}^{2}$ \\ ${ }^{1}$ Bejing Institute of Graphic Communication \\ ${ }^{1}$ Beijing Key Laboratory of Signal and Information Processing for High-End Printing Equipment, Beijing Institute of Graphic \\ Communication
}

\begin{abstract}
In order to optimize the financial management process and improve the working efficiency of printing enterprise, a financial accounting module for bills is designed and realized. The web interface of system is realized through the technology of Java and JavaScript, adopting the development mode of browser and server $(\mathrm{B} / \mathrm{S})$, all information about bills are stored with SQL Server database. There are three functions including implementing, invoicing for bills, management for receipt and distribution of money for all invoices and receipts.
\end{abstract}

\section{INTRODUCTION}

With the development of enterprise and expansion of business scale, the financial problems are more serious. The process is complicated and easy to bring out trouble in traditional manual bookkeeping. Therefore, financial management plays a more important role in business scale. At present, manual accounting method is accepted in most of small and medium-size enterprise, the result of this is accumulation of paper bills and loss of important data. The worker will waste more time to organize bills, efficiency is lower [1].

Financial accounting refers to the management for different invoice and receipt in enterprise, which can effectively help enterprise meet the requirements of modern financial management. Compared with the traditional manual accounting method, the informatization and automation of financial work are the main advantages, in other words, the system can automatically complete the financial accounting according to the information of original vouchers. Therefore, it can achieve standardized management of financial information and strengthen the confidentiality and reliability of financial information [2].

In the enterprise's capital accounting, there are usually more bills problems. Based on these problems, the financial accounting module for bills are designed and implemented in this paper.

\section{Demand analysis}

This paper takes the accounting of bills in financial management as an example to illustrate the process from the issue of an invoice to the completion of collection and the related data is stored in the SQL Server database. The implemented functions included:

(1) Invoice is generated for different bills to realize the maintenance and checking of invoice information.

(2) Receipt is generated for checking invoice to realize the maintenance and checking of the collection information.

(3) The money of invoice and receipt is distributed automatically, when finished, the relevant information is stored in the bill table.

This paper introduces UML use case on the systems function analysis, shown in Figure.1.

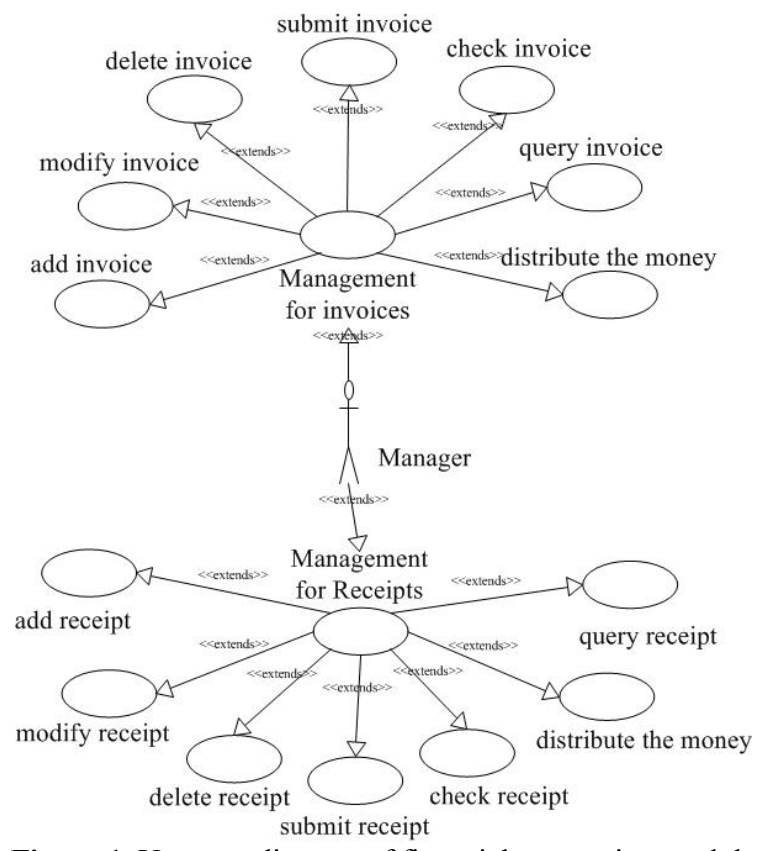

Figure 1. Use case diagram of financial accounting module 


\section{System design}

\subsection{Design of the main functional modules}

The system includes two functional modules: invoice management and receipt management.

(1) The information contained in the invoice mainly comes from the checked bill. When an invoice is added, the system automatically selects the bill from the customer according to the its name entered in the bill, which not only facilitates the improvement of the billing information, but also reduces the time required for the staff to find the bills with the same client and improve the work efficiency.

The total invoice money will be automatically assigned according to the order of the bills. After the invoice is passed, the bills whose money are the same as the invoice money will no longer appear in the added list.

(2) When an invoice is approved, it can be used to certify that productive task have finished, then a receipt can be added to manage collection information. Due to the collection cannot be finished at one time, so the numbers of receipt is not limited, and the full receipt money will be automatically assigned to bills.

(3) All the money of approved invoices and receipts will be automatically written into the bills as subsequent audits.

The specific process is shown in Figure. 2.

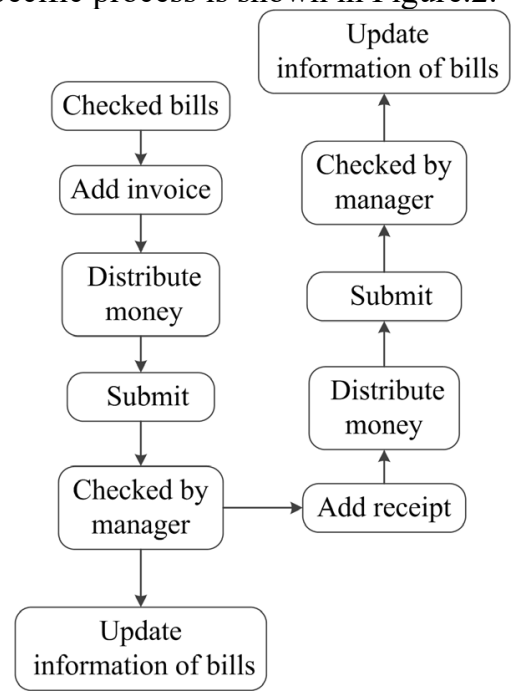

Figure 2. The financial process

\subsection{Design of database}

The basic function is to solve the analysis and processing of complex data information in enterprise information system, and database design is the foundation to establish it. It is responsible for providing appropriate procedures for data processing and storage [3]. Because the system involves two types of documents, and both types of documents have information that needs to be rewritten after the audit is completed, the database tables with different names and fields are designed according to different requirements. The structure and relationship between database tables are shown in Figure.4.

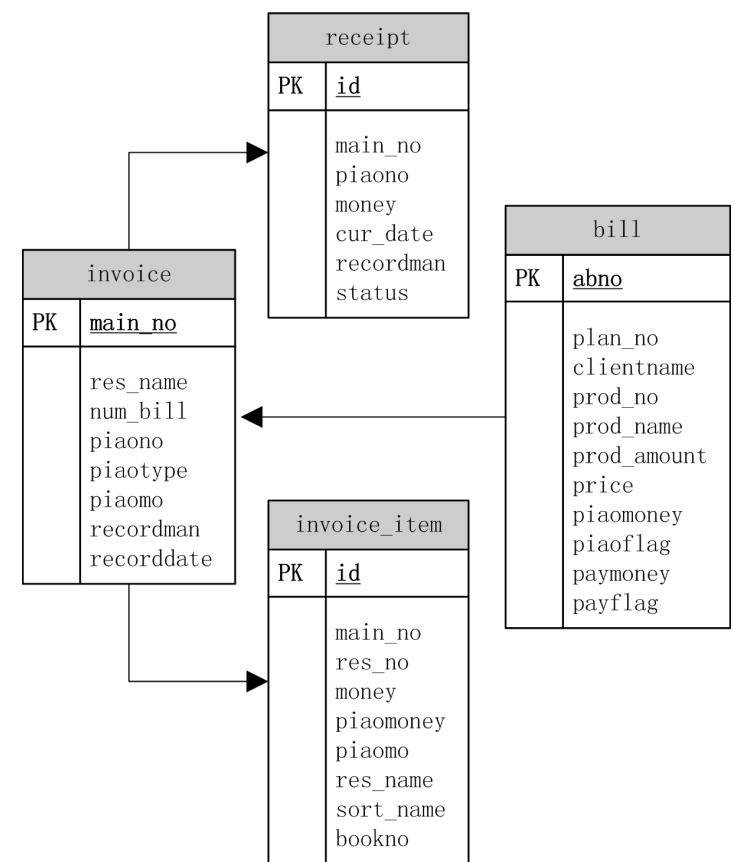

Figure 4. Financial management table structure

There are four tables listed by the field of main no in the database. The invoice management refers to two tables: invoice and invoice item, including the invoice number, source enterprise, bill number, invoice type, total money, etc. The receipt management refers to one table: receipt, including the invoice number, collection amount, collection date and other information. When the information in tables of invoice and receipt is modified, relevant information in bill table will be updated.

\section{System implementation}

\subsection{System implementation environment and technology}

In the process of system development, the platform of Dorado7[5] is selected. Dorado7 based on the eclipse is used as a system development tool. The testing environment is window OS. More development environment is as shown in Table 1.

Table 1. Development environment

\begin{tabular}{|l|l|}
\hline Demand name & Platform and tools \\
\hline Hardware platform & Computer \\
\hline Development tool & Eclipse \\
\hline Development language & Java, JavaScript \\
\hline Compile environment & Win7+JDK \\
\hline Testing environment & Win7+Chrome \\
\hline Data base & SQL Server \\
\hline
\end{tabular}

Three-tier structure of browser/server $(\mathrm{B} / \mathrm{S})$ is used in system development, including the interface layer, 
application layer and data layer. During the development process, the financial accounting module is developed on the Eclipse platform using the Java language, and the software can be implemented on different platforms such as Windows, UNIX and Linux. Data information is managed by SQL Server database, it is a relational database management system, which integrates various types of Microsoft products and resources, provides a powerful visual interface, and has great significance in the rapid development of enterprise systems [4].
The financial accounting module designed in this paper is mainly applicable to small and medium-sized printing companies. After receiving orders, systems will generate bills based on the materials, equipment and labour needed for production, including the total money of production, packaging and delivery. The finance department will issue invoice vouchers for all bills for the same customer, as well as the receipt vouchers for each invoice after the production task is completed, financial accounting sequence diagram design shown in Figure.5.

\subsection{Business process implementation of financial accounting}

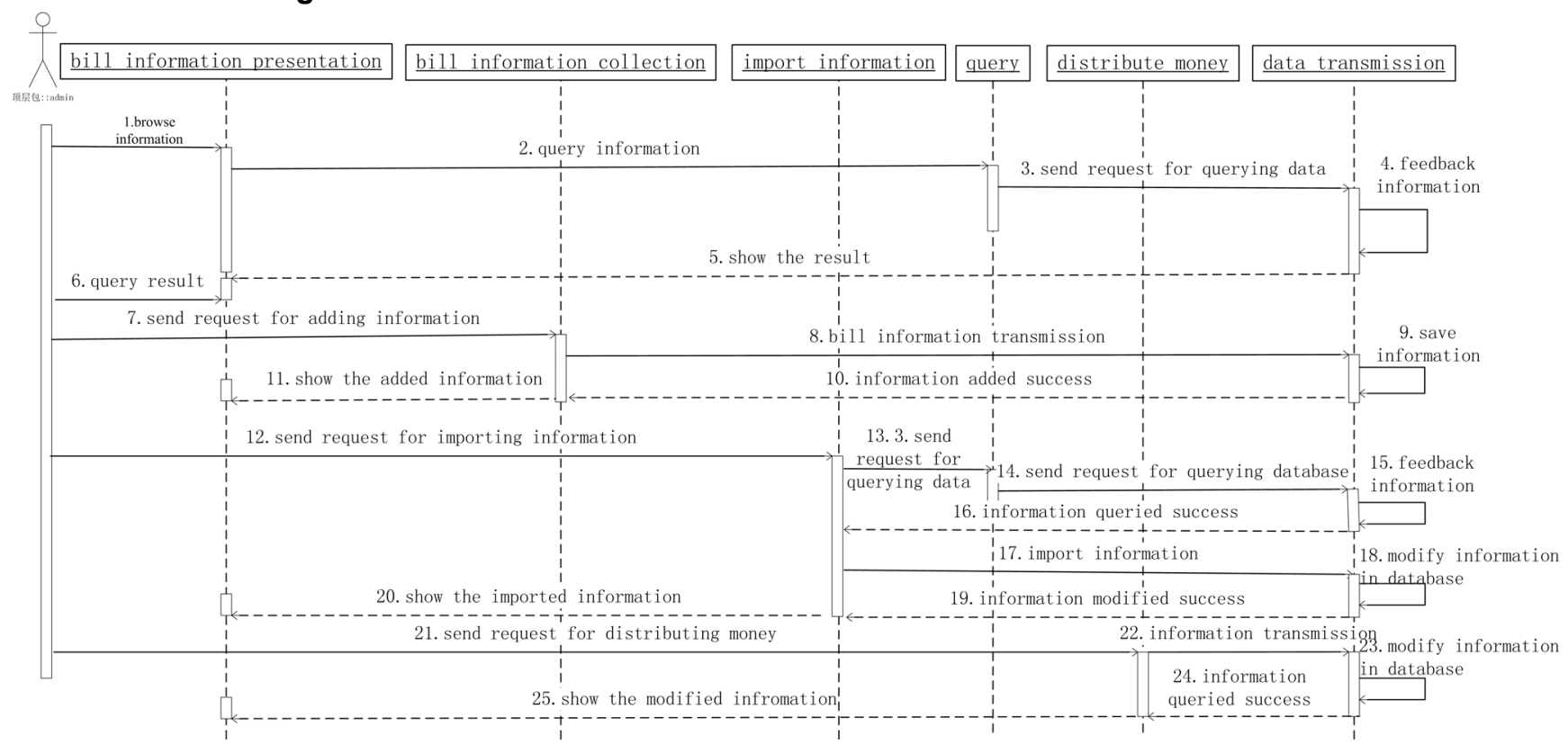

Figure 5. Financial accounting sequence diagram design

The specific implementation steps as follows:

(1) Generate qualified bills, which is a prerequisite for financial accounting. The system generates a bill based on the work order information provided by the business department, which includes the cost of materials required for production, packaging, as well as labour costs and transportation expenses required for delivery, that is, the total amount the customer needs to pay. At the same time, there may be multiple work orders from the same customer in the work order information base, and the system will generate different bills for this situation.

(2) Generate an invoice. After the production task is completed, the finance department opens an invoice, which is the receipt certificate. When the invoice is opened, the system will automatically add all the bill information of the customer to the invoice according to the customer information of the bill, and at the same time, the total money of the invoice will be automatically allocated to each invoice. When the invoice money allocated is the same as the bill money, it indicates that the bill is invoiced. If it is inconsistent, it will be redistributed when the invoice is reissued.

(3) Generate a receipt. Only the invoice has been completed and checked by manager, it can be added receipts. The total money of the receipt is also automatically allocated to each bill. When the money of receipt is the same as the bill money, the receipt is completed.

The main interface for system is shown in Figure.6, including two images and used to introduce the process of creating a receipt. 


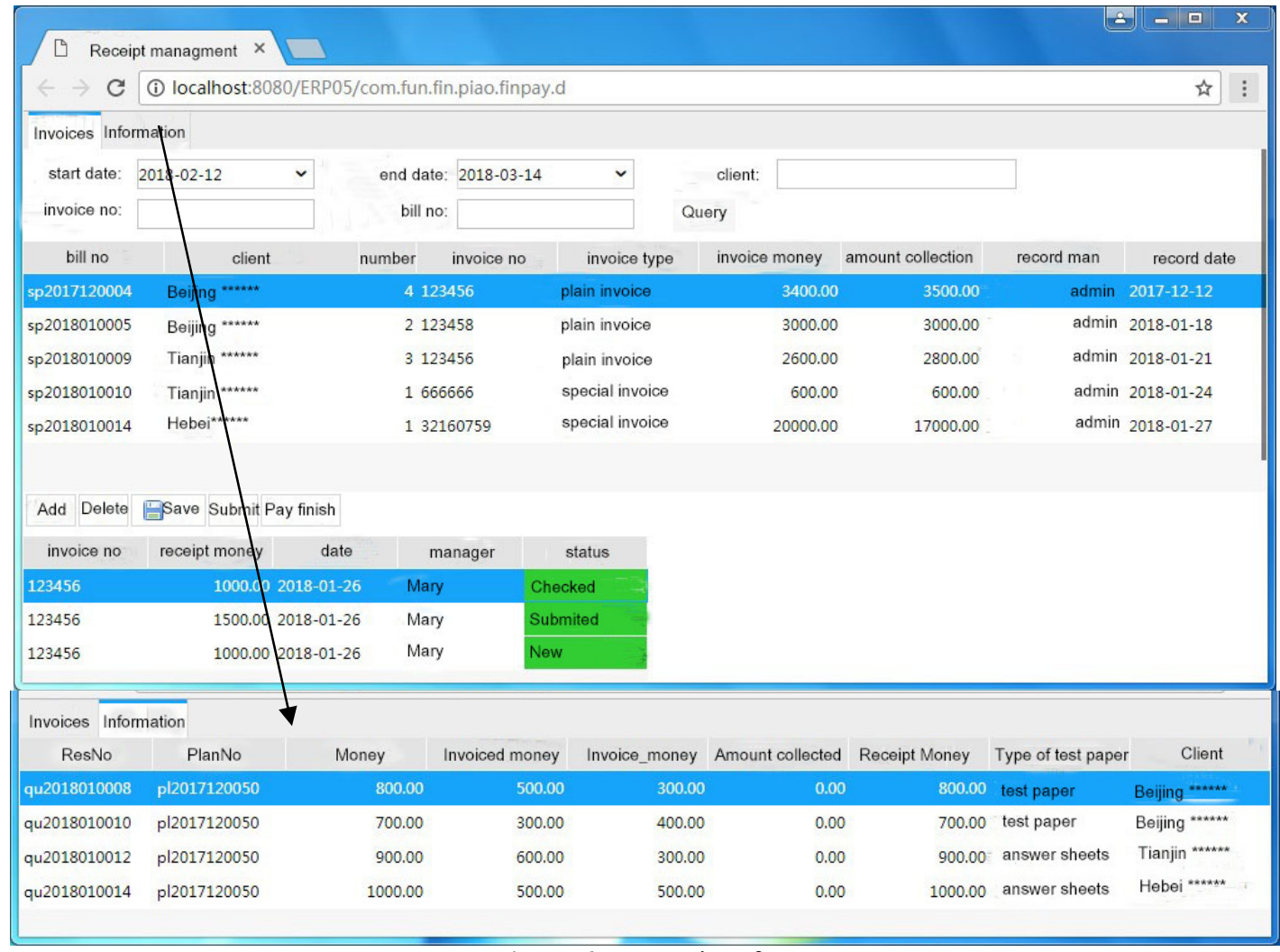

Figure 6. System interface

There are two parts in the first image. The upper parts contains the main information of invoices, all the invoices are checked by manager, in other words, only checking invoice can be added receipt. All information about invoice can be browsed by user, but can't be modified. User can obtain required invoice by means of setting different conditions, including the date, client, invoice number and bill number. The nether parts contains the main information of receipts. Any invoice shown in the upper parts can be added one or more receipts, then the detail information can be added in the created receipt, all the information can be stored in database. If the receipt has no mistakes, it can be submitted for manager to check, or completed the process of collection, relevant information in bill table will be updated.

The detail information of invoice is shown in the second image, including the resource number, plan number and the detail income and expenditure.

\section{Conclusion}

The financial accounting module based on web is designed and realized, which can generate invoices for different bills, and generate receipts for invoices that have been checked. And the functions of maintaining and checking invoice information and receipt information also are realized. At the same time, the invoice money and the receipt money are automatically allocated to the bill. The implemented system is suitable for small and medium-sized printing enterprise, which can improve the financial management level of enterprises, increase the work efficiency of staff members, and achieve effective management and storage of enterprise information, thereby strengthening financial supervision and providing better decision support for management.

\section{Acknowledgment}

The paper is supported by National Natural Science "Nonlinear Dynamics model and Analysis of Gear Systems for Offset Press" (No.61472461).

\section{References}

1. Xue Wang. Design and Implementation of Enterprise Financial Management System [D].Jilin: Jilin University, 2016.

2. Ling Jian. Design and Implementation of Enterprise Financial Management System Based on Web [D]. Sichuan: University of Electronic Science and Technology of China, 2012.

3. Meijuan Liao. Design and Implementation of ERP System for Small and Medium Sized Printing Enterprise [D]. Zhejiang : Zhejing University of Technology, 2016.

4. Tomorrow's technolog. SQL Server from the beginning to the mastery $[\mathrm{M}]$. Beijing : Tsinghua University Press, 2012.

5. Dorado7 database[EB/OL]. http://wiki.bsdn.org/display/dorado7/Project+Home 